Journal of

Cutaneous Pathology

ISSN 0303-6987

\title{
Antibody to $\$ 100$ a6 protein is a sensitive immunohistochemical marker for neurothekeoma
}

Background: Neurothekeoma is a benign tumor of putative peripheral nerve sheath origin. It occurs in a myxoid (classic) variant, cellular variant, and intermediate (mixed) variant. Cellular neurothekeoma (CNT) usually involves the head and neck or extremities of young patients. Histologically, GNTcan be confused with melanocytic and fibrohistiocytic lesions. An immunohistochemical antibody panel is often necessary to confirm the histological impression and exclude melanocytic and/or fibrohistiocytic lesions.

Methods: Formalin-fixed, paraffin-embedded archival tissues were evaluated by immunohistochemistry using antibodies specific for S100A6 and PGP9.5 in 11 cases of neurothekeoma (seven cellular, four myxoid). A variety of other antibodies were evaluated by immunohistochemistry at the time of initial diagnosis.

Results: All 11 neurothekeoma cases were positive for S100A6 protein (four cases, weak $/ 1+$; seven cases, strong $/ 2+$ ), corresponding to $100 \%$ sensitivity. In contrast, eight of 11 neurothekeoma cases (73\% sensitivity) were positive for PGP9.5. All seven CNTcases were negative for S100B, as expected.

Conclusions: Anti-S100A6 is a highly sensitive antibody for neurothekeomas, including CNT, and, in our experience, is superior in sensitivity to PGP9.5. However, like other antibodies used in evaluating neurothekeomas, S100A6 lacks specificity, as has been demonstrated in previous studies. Nevertheless, S100A6 can be useful in an immunohistochemical antibody panel to evaluate lesions where the differential diagnosis includes CNT.

Fullen DR, Lowe L, Su LD. Antibody to S100a6 protein is a sensitive immunohistochemical marker for neurothekeoma.

J Cutan Pathol 2003; 30: 118-122. (C) Blackwell Munksgaard 2003.

\section{Douglas R. Fullen, Lori Lowe and Lyndon D. Su}

${ }^{1}$ Departments of Pathology and Dermatology, University of Michigan Hospitals, Ann Arbor, Michigan, MI, USA
Douglas R. Fullen, MD, Department of Pathology, University of Michigan Hospitals, M5230, Medical Sciences I, 1301 Catherine, Ann Arbor, MI 48109-0602, USA

Tel: (734) $764-4460$

Fax: (734) 936-2756

e-mail:dfullen@umich.edu

Accepted September 10, 2002
Neurothekeoma was originally described in 1969 by Harkin and Reed under the appellation of nerve sheath myxoma. ${ }^{1}$ This benign, dermal-based tumor of putative peripheral nerve sheath origin was subsequently coined neurothekeoma in 1980 by Gallager and Helwig, based on their observations in a series of 53 cases. $^{2}$ Their tumors had a predilection for the central region of the face, shoulders and arms of predominantly young females. Histologically, their lesions were composed of variable proportions of epithelioid and spindle cells in a variably mucinous stroma.
In 1986, Rosati et al. proffered the term cellular neurothekeoma for the more cellular variant of neurothekeoma. ${ }^{3}$ There are currently three subtypes of neurothekeoma recognized, based on cellularity of the tumor, growth pattern, and amount of stromal mucin. ${ }^{4}$ The hypocellular or classic (myxoid) type has a circumscribed, distinctly lobulated growth pattern, with low cellularity and abundant myxoid stroma. The cellular type is more ill defined, fascicular in its growth pattern, hypercellular, and possesses little to scant stromal mucin. The mixed type demonstrates 
intermediate features between the classic (myxoid) and cellular types.

While the diagnosis of myxoid neurothekeoma (MNT) is usually not problematic, the same can not be said for cellular neurothekeoma (CNT). Cases of CNToften histologically resemble melanocytic lesions, such as Spitz nevus or melanoma, smooth muscle tumor, or fibrohistiocytic lesions. ${ }^{5-8}$ In addition, it is quite common for CNT to demonstrate atypical features, including low-grade cytologic atypia and mitotic figures, further contributing to its confusion with melanoma or an atypical Spitz lesion. ${ }^{9}$ Therefore, these lesions are often submitted for dermatopathology consultation for definitive diagnosis. To this end, a panel of immunohistochemic markers is often necessary to rule out a melanocytic or occasionally fibrohistiocytic lesion. By immunohistochemistry, CNT typically lacks expression of S100 protein, as well as other markers of nerve sheath differentiation (NGFR, GFAP and CD57); moreover, CNT lacks expression of more specific melanocytic markers, such as HMB-45. ${ }^{4,9-12}$ Recently, protein gene product (PGP)9.5, a protein with broad expression in neural tissues, has been reported as a useful marker for cellular neurothekeoma in an immunohistochemical panel. ${ }^{13}$

Additional immunohistochemical markers may be helpful in an antibody panel for evaluation of CNTs. S100A6 protein or calcyclin, a member of the S100 protein superfamily, has been isolated from a variety of normal cell types, including: melanocytes, Schwann and Schwann-like cells, Langerhans'cells, dermal dendrocytes, some glandular epithelium, and keratinocytes (weak). ${ }^{14-16}$ Antibody to this protein has been shown to label several tumors characterized by the presence of many Schwann cells, such as Schwannomas, neurofibromas, and solitary circumscribed (palisaded encapsulated) neuromas. ${ }^{17}$

We studied S100A6 protein expression in a series of 11 neurothekeomas (both CNT and MNT) to determine if this antibody was a useful marker for this tumor and compared it with our results for PGP9.5.

\section{Materials and methods}

After obtaining approval from the University of Michigan Medical Institutional Review Board, 11 cases of neurothekeoma, seven CNT and four MNT were retrieved from the Dermatopathology Consult Service and Archives of Surgical Pathology in the Pathology Department at the University of Michigan. The diagnoses were based on standard published histopathologic criteria, ${ }^{11}$ and results of immunohistochemical stains at the time of diagnosis.

Formalin-fixed, paraffin-embedded tissue sections, approximately $5-\mu$ thick, were placed on charged (plus) slides. The sections were deparaffinized and rehydrated. Endogenous peroxidase activity was quenched with $3 \% \mathrm{H}_{2} \mathrm{O}_{2}$ in distilled water for $10 \mathrm{~min}$. Antigen retrieval was accomplished by pretreatment in proteinase $\mathrm{K}$ for $20 \mathrm{~min}$ for anti-S100A6 and pretreatment in $10 \mathrm{mM}$ of citrate buffer $(\mathrm{pH} 6.0)$ in a microwave pressure cooker for $10 \mathrm{~min}$ for antiPGP9.5. Primary antibodies to S100A6 protein/clone CACY-100 (1: 500 dilution, mouse monoclonal, Sigma, St. Louis, MO, USA) and PGP9.5 (1:200 dilution, rabbit polyclonal, Dako, Carpinteria, CA, USA) were applied to all cases. Bound primary antibodies were detected using an avidin-biotin peroxidase system. The antigen-antibody complexes were localized with $3,3^{\prime}$ diaminobenzidine (DAB). The slides were counterstained with weak Harris' hematoxylin.

Antibody to S100B was performed at the time of diagnosis in all cases and was available for review. In addition, antibodies to HMB-45, Melan-A (MART-1), NK1-C3, cytokeratins (AE1/AE3 and CAM5.2 mixture), epithelialmembraneantigen(EMA), carcinoembryonic antigen (CEA), chromogranin A, neuron specific enolase (NSE), vimentin, CD68, smooth muscle actin (SMA), CD34, factor XIIIa, and CD31 were performed at the time of diagnosis in select cases and were also available for review. Antibodies to S100A6 protein and PGP9.5 were not performed on any cases at the time of initial diagnosis.

Immunoperoxidase stains were evaluated by two of the authors (LDS and DRF). Immunolabeling was judged to be positive [weak/focal $(1+)$ or strong $(2+)$ ] or negative for S100A6 and PGP9.5. Additional antibodies performed at the time of the initial diagnosis were simply reported as positive or negative and not semiquantitated. Positive and negative controls were appropriate for each antibody run.

\section{Results}

\section{Clinical features}

In our series, neurothekeomas involved relatively young patients (overall mean age 25.8 years; range 13-43 years), with a predilection for younger patients with CNT (mean age, 20.9 years) compared with those with MNT (mean age, 34.5 years). Of the 11 patients, six were males and five were females. The lesions were most commonly located on the head and neck (54\%, six cases), followed by the extremities [ $36 \%$, four cases total: three cases (upper extremity), one case (lower extremity)] and trunk (9\%, one case, chest). Six of seven cases of CNT involved the head and neck, whereas all four MNT involved the extremities.

\section{Histopathologic features}

All 11 cases of neurothekeoma were dermal-based tumors that lacked epidermal involvement. Cases of MNT were characterized by well-circumscribed 


\section{Fullen et al.}

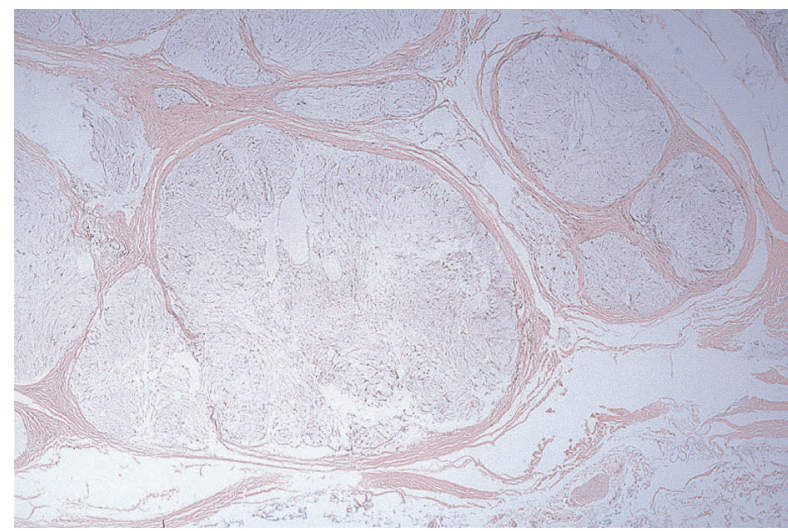

Fig. 1. Lobular architecture of a myxoid neurothekeoma.

lobules composed of spindle cells, loosely arranged and in whorls, within abundant myxoid stroma, and were separated from the adjacent dermis by thin fibrous septa (Fig. 1). Cases of CNT were ill-defined, less circumscribed tumors than MNT cases. These tumors formed cellular fascicles and nests of predominantly epithelioid cells with variable amounts of eosinophilic to pale cytoplasm (Fig. 2). Scant myxoid stroma was present in most cases. Occasional mitotic figures and low-grade cytologic atypia were observed in most cases.

\section{Immunohistochemistry}

All cases of neurothekeoma demonstrated positive labeling of tumor cells with anti-S100A6 [weak $(1+)$, four cases; strong $(2+)$, seven cases]. With respect to the CNT cases, three of seven cases demonstrated strong (2+) anti-S100A6 labeling, and four of seven cases demonstrated weak $(1+)$ anti-S100A6 labeling (Fig. 3). All four cases of MNT showed strong $(2+)$ anti-S100A6 labeling of spindle cells without diffuse staining of the myxoid stroma, a feature commonly observed with anti-S100B (Fig. 4).

PGP9.5 was detected in eight of 11 (73\%) cases of neurothekeoma. Three of seven cases of CNT were negative

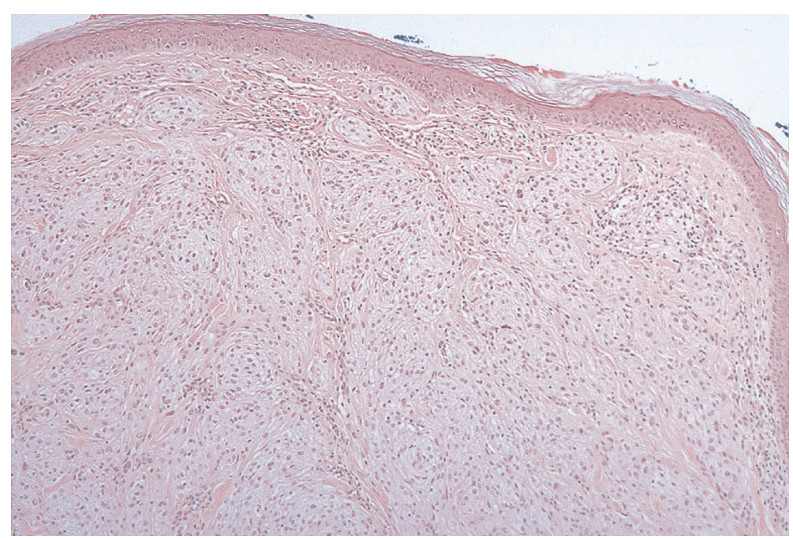

Fig. 2. Nests and fascicles of predominantly epithelioid cells in the dermis in a cellular neurothekeoma.

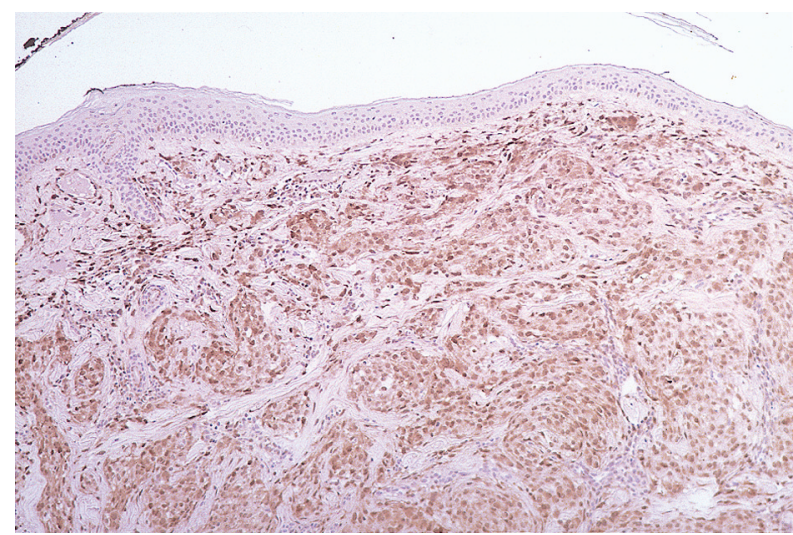

Fig. 3. S100A6 protein expression in a cellular neurothekeoma.

for PGP9.5 (Fig. 5); all three PGP9.5-negative cases were strongly $(2+)$ positive for S100A6 protein and two cases were also positive for vimentin. All four cases of MNT had weak $(1+)$ labeling of spindle cells for PGP9.5.

S100B protein was present in only three of 10 neurothekeomas, all MNTcases; one MNTwas not evaluated for S100B protein at the time of diagnosis. All seven cases of CNTwere negative for S100B protein (Fig. 6).

Select cases of CNT were also evaluated for a variety of other immunohistochemical markers at the time of
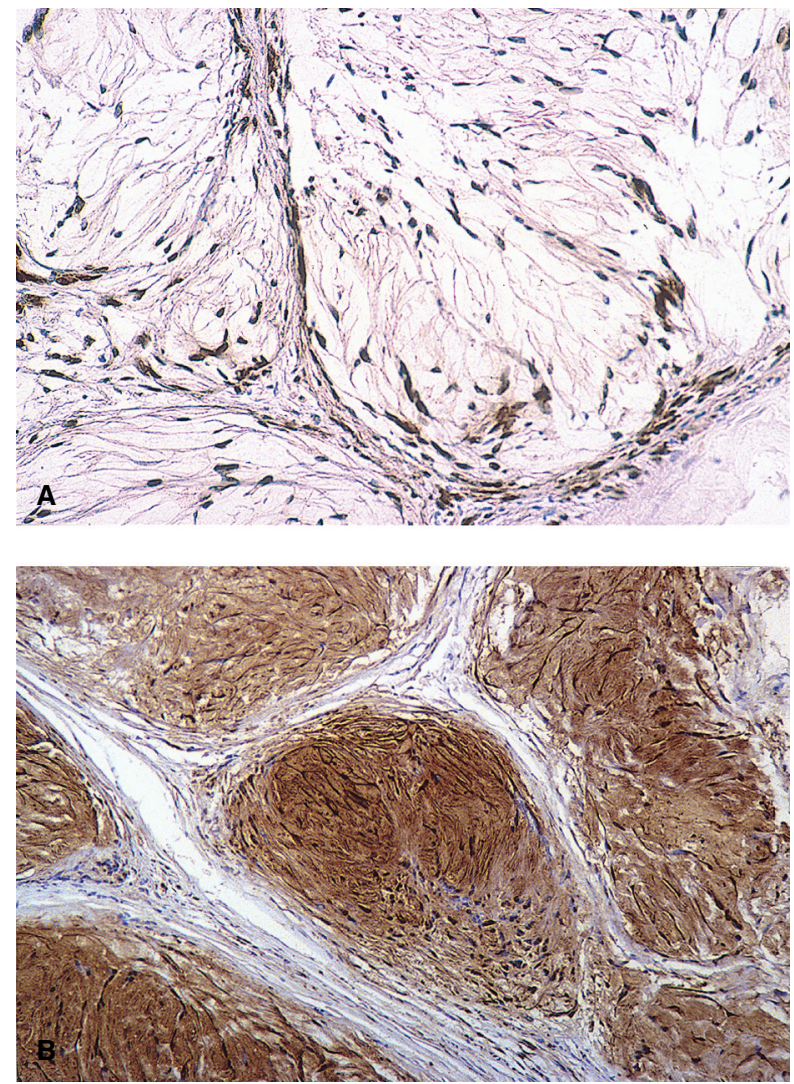

Fig. 4. Expression of S100 proteins in a myxoid neurothekeoma. (A) S100A6 protein and (B) S100B protein. Note the diffusion of the S100B protein into the myxoid matrix. 
S100A6 protein in neurothekeomas
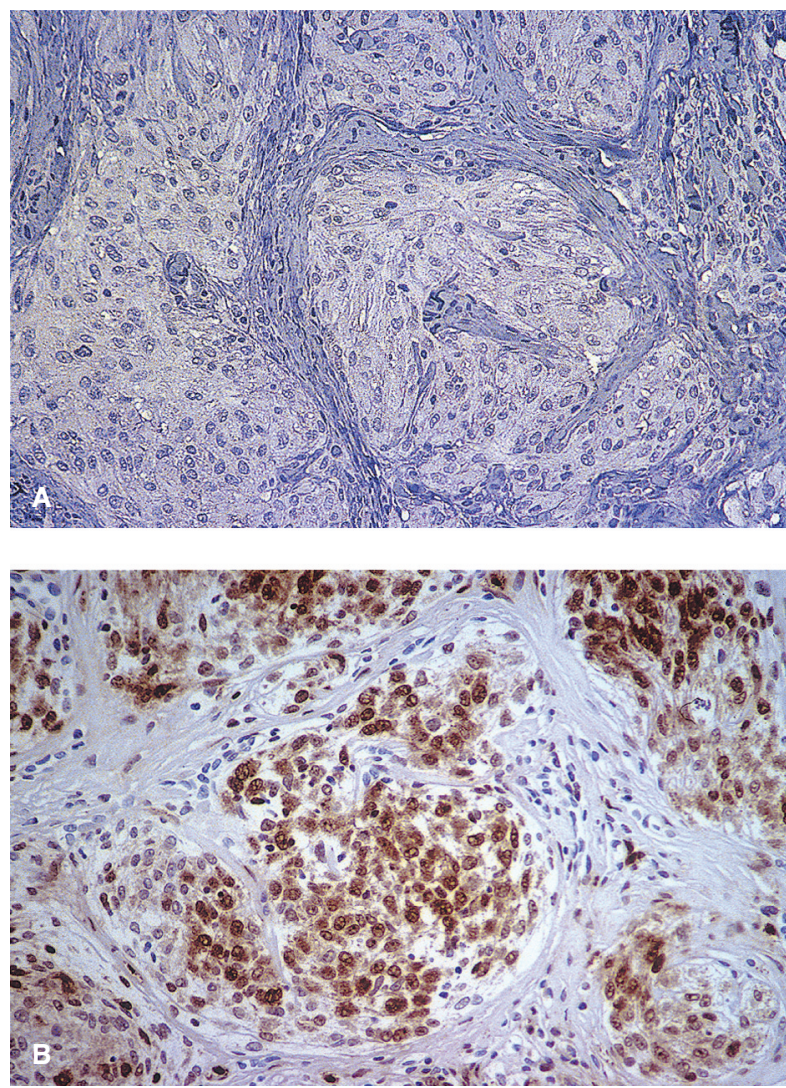

Fig. 5. Example of a cellular neurothekeoma that was (A) negative for PGP9.5 and (B) positive for S100A6 protein.

diagnosis. Cellular neurothekeomas were positive for vimentin (three cases), NK1-C3 (one case), and NSE (one case). Cellular neurothekeomas were negative for melan-A/MART-1 (five cases), HMB-45 (three cases), cytokeratins (three cases), CD68 (three cases), SMA (two cases), CD34 (two cases), EMA (one case), CEA (one case), chromogranin A (one case), and CD31 (one case). The results of the immunohistochemical stains performed on the CNTs are summarized inTable 1 .

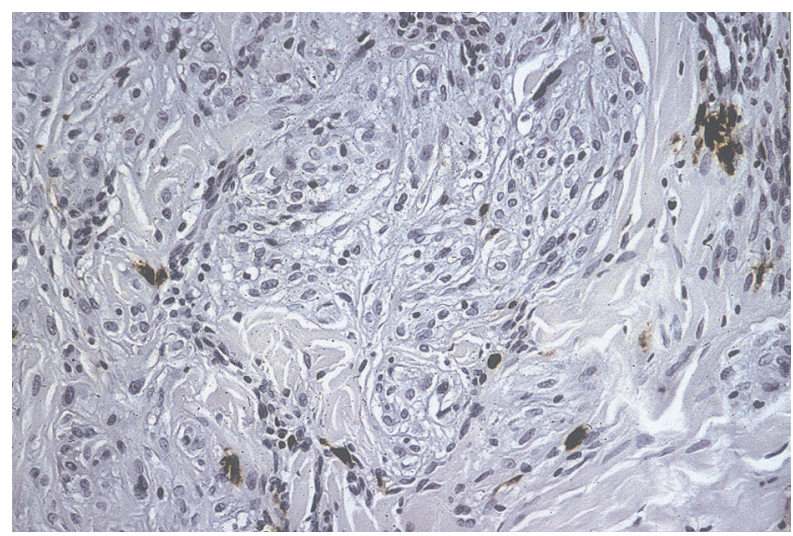

Fig. 6. Negative S100B labeling in tumor cells of a cellular neurothekeoma. Note the entrapped S100B-positive dendritic cells in the tumor.
Table 1. Results of immunohistochemic stains in cellular neurothekeomas

\begin{tabular}{|c|c|c|c|c|}
\hline Patient no. & S100A6 & PGP9.5 & $\mathrm{S} 10 \mathrm{C}$ & Other \\
\hline 1 & ++ & + & - & $\begin{array}{l}\operatorname{Vim}(-), \operatorname{NSE}(-), \text { EMA }(-) \\
\text { Melan-A }(-)\end{array}$ \\
\hline 2 & + & + & - & ND \\
\hline 3 & + & + & - & $\begin{array}{l}\text { NK1-C3 }(+), \text { Melan-A (-), } \\
\text { HMB-45 (-) }\end{array}$ \\
\hline 4 & ++ & - & - & $\begin{array}{l}\text { Vim }(+), \text { Melan-A }(-) \\
\text { CD68 }(-), \text { SMA }(-)\end{array}$ \\
\hline 5 & ++ & - & - & $\begin{array}{l}\operatorname{Vim}(+), \operatorname{HMB}-45(-), \mathrm{CK}(-), \\
\text { CD34 }(-), \operatorname{CD} 31(-)\end{array}$ \\
\hline 6 & + & + & - & Melan-A (-), Factor XIIIa (-) \\
\hline 7 & ++ & + & - & ND \\
\hline
\end{tabular}

Vim, vimentin; NSE, neuron-specific enolase; EMA, epithelial membrane antigen; SMA, smooth muscle actin; CK, cytokeratins; ND, not done.

\section{Discussion}

The clinical and histopathologic findings in our cases of neurothekeoma were similar to previous published series. ${ }^{1,4-6,9,11,13}$ Our lesions had a predilection for the head and neck of young patients, as is quite typical, although our MNT cases were all extremity based lesions.

A diagnosis of MNT is usually not problematic, but the same can not be said for all CNTcases. Often, as has been our experience, CNTare referred for consultation because of difficulties distinguishing this lesion from a melanocytic lesion, such as a (atypical) Spitz nevus, cellular blue nevus or melanoma, or a fibrohistiocytic lesion. Obviously, these distinctions are important for treatment decisions, especially with respect to melanocytic lesions.

The absence of S100B protein expression in CNT is well documented in the literature $;^{3-7,9-13}$ however, it is difficult to make a conclusive diagnosis based on a negative immunohistochemical staining result. For this reason, it is usually necessary to perform a panel of immunohistochemical markers, and render a diagnosis based on the overall immunoreactivity profile of the tumor cells. There are few antibodies that are consistently expressed by tumor cells in CNT. Calonje et al. published a series of nine cases that were all positive for NK1-C33. ${ }^{6}$ However, NK1-C3 has been demonstrated in a wide spectrum of lesions, such as a variety of melanocytic nevi, melanoma, granular cell tumors, medullary carcinoma of the thyroid gland, carcinoid tumors, carcinomas of the breast and prostate gland, and some fibrohistiocytic lesions. ${ }^{18-23}$ Within the past few years, Wang et al. showed PGP9.5 expression in all 19 of their neurothekeoma cases, including $12 \mathrm{CNT}^{13}$ Unfortunately, anti-PGP9.5 suffers from low specificity and can be positive in a variety of tumors of neuroectodermal origin. ${ }^{24,25}$

Based on previous experience with S100A6 protein expression in tumors of putative Schwann cell origin, ${ }^{17}$ we wondered whether S100A6 protein would be 
expressed in neurothekeomas, especially CNT. Moreover, we were interested in comparing anti-S100A6 with anti-PGP9.5 in these tumors in our institution. We demonstrated that all 11 of our neurothekeoma cases were positive for S100A6 (100\% sensitivity), but only eight of $11(73 \%)$ neurothekeomas (five of seven CNT) stained for PGP9.5. Thus, in our experience, anti-S100A6 was superior in sensitivity to antiPGP9.5. Perhaps our lower sensitivity for PGP9.5 compared with Wang et al. ${ }^{13}$ was the result of using a primary antibody from a different manufacturer, i.e. our antibody was obtained from Dako and theirs from Biogenesis. We used an antigen-retrieval method similar to Wang et al. ${ }^{13}$

Anti-S100A6 suffers from a similar lack of specificity to anti-PGP9.5 and anti-NK1-C3. In addition to tumors of Schwann cell differentiation, S100A6 is also expressed in some melanocytic and fibrohistiocytic lesions. ${ }^{14,26}$ In fibrohistiocytic lesions, S100A6 protein and factor XIIIa usually shows the same labeling pattern; therefore, a tumor that is positive for S100A6 and negative for factor XIIIa is very unlikely to be of fibrohistiocytic lineage. ${ }^{26}$ In melanocytic lesions, it has previously been demonstrated that benign and malignant melanocytic lesions consistently demonstrate both S100B and S100A6 expression; therefore, a lesion that is positive for S100A6 and negative for S100B is very unlikely to be of melanocytic lineage.

In conclusion, antibody to S100A6 protein, as a result of its lack of specificity, can not be used alone in the evaluation of CNT. However, anti-S100A6 is a highly sensitive immunohistochemical marker for GNT, which can be used in a panel of antibodies that exclude melanocytic lesions and, if necessary, fibrohistiocytic lesions. Moreover, S100A6 protein expression in CNTdoes not shed additional light on the histogenesis of this tumor, but does not refute an origin from the peripheral nerve sheath.

\section{References}

1. Gallager RL, Helwig EB. Neurothekeoma - A benign cutaneous tumor of neural origin. AmJ Clin Path 1980; 74: 759.

2. Harkin JC, Reed RJ. Tumors of the peripheral nervous system. In: RosaiJ, Sobin LH (eds). Fascicle 3, 2nd edn. Washington DC: Armed Forces Institute of Pathology, 1969; 60.

3. Rosati LA, Fratamico FGM, Eusebi V. Cellular neurothekeoma. Appl Pathol 1986; 4: 186.

4. Argenyi ZB, LeBoit PE, Santa Cruz D, Swanson PE, Kutzner H. Nerve sheath myxoma (neurothekeoma) of the skin: light microscopic and immunohistochemical reappraisal of the cellular variant. J Cutan Pathol 1993; 20: 294.

5. Barnhill RL, Mihm MC. Cellular neurothekeoma: a distinctive variant of neurothekeoma mimicking nevomelanocytic tumors. AmJ Surg Pathol 1990; 14: 113.

6. Calonje E, Wilson-Jones E, Smith NP, Fletcher CDM. Cellular 'neurothekeoma': an epithelioid variant of pilar leiomyoma?
Morphological and immunohistochemical analysis of a series. Histopathology 1992; 20: 397.

7. Husain S, Silvers DN, Halperin AJ, McNutt NS. Histologic spectrum of neurothekeoma and the value of immunoperoxidase staining for S100 protein in distinguishing it from melanoma. AmJ Dermatopathol 1994; 16: 496.

8. Zelger BG, Steiner H, Kutzner H, Maier H, Zelger B. Cellular 'neurothekeoma': An epithelioid variant of dermatofibroma? Histopathology 1998; 32: 414.

9. Busam KJ, MentzelT, Colpaert C, Barnhill RL, Fletcher CDM. Atypical or worrisome features in cellular neurothekeoma. A study of 10 cases. AmJ Surg Pathol 1998; 22: 1067.

10. Argenyi ZB, Kutzner H, Seaba MM. Ultrastructural spectrum of cutaneous nerve sheath myxoma/cellular neurothekeoma. J Cutan Pathol 1995; 22: 137.

11. Barnhill RL, Dickersin GR, Nickeleit V et al. Studies on the cellular origin of neurothekeoma: Clinical, light microscopic, immunohistochemical, and ultrastructural observations. J Am Acad Dermatol 1991; 25: 80.

12. Tomasini C, Aloi F, Pippione M. Cellular neurothekeoma. Dermatology 1996; 192: 160.

13. Wang AR, May D, Bourne P, Scott G. PGP. 9.5: a marker for cellular neurothekeoma. AmJ Surg Pathol 1999; 23: 1401.

14. Böni R, Burg G, Doguoglu A et al. Immunohistochemical localization of the $\mathrm{Ca} 2+$ binding S100 proteins in normal human skin and melanocytic lesions. BrJ Dermatol 1997; 137: 39.

15. KuźnickiJ, Filipek A, Heimann P, Kaczmarek L, Kamińska B. Tissue-specific distribution of calcyclin $10.5 \mathrm{kDa}$ Ca2+-binding protein. FEBS Lett 1989; 254: 141.

16. Tonini GP, Casalaro A, Cara A, DiMartino D. Inducible expression of calcyclin, a gene with strong homology to S-100 protein, during neuroblastoma cell differentiation and its prevalent expression in Schwann-like cells. Cancer Res 1991;51:1733.

17. Fullen DR, ReedJA, Finnerty B, McNutt NS. S100A6 preferentially labels type $\mathrm{C}$ nevus cells and nevic corpuscles: Additional support for Schwannian differentiation of intradermal nevi. J Cutan Pathol 2001; 28: 393.

18. Mackie RM, Campbell I, Turbitt ML. Use of NK1 C3 monoclonal antibody in the assessment of benign and malignant melanocytic lesions. J Clin Pathol 1984; 37: 367.

19. Vennegoor G, CalafatJ, Hageman P et al. Biochemical characterization and cellular localization of a formalin-resistant melanoma-associated antigen reacting with monoclonal antibody NK1-C3. Int J Cancer 1985; 35: 287.

20. Gatter KC, Ralfkiaer E, SkinnerJ et al. An immunocytochemical study of malignant melanoma and its differential diagnosis from other malignant tumours. J Clin Pathol 1985; 38: 1353.

21. Mahalingam M, LoPiccolo D, Byers HR. Expression of PGP9. 5 in granular cell nerve sheath tumors: an immunohistochemical study of six cases. J Cutan Pathol 2001; 28: 282.

22. Zelger BW, Zelger BG, Steiner H, Ofner D. Aneurysmal and hemangiopericytoma-like fibrous histiocytoma. J Clin Pathol 1996; 49: 313.

23. Zelger BG, Sidoroff A, Zelger B. Combined dermatofibroma: coexistence of two or more variant patterns in a single lesion. Histopathology 2000; 36: 529.

24. Rode J, Dhillon AP, Doran JF, Jackson P, Thompson RJ. PGP. 9.5-a new marker for human neuroendocrine tumors. Histopathology 1985; 9: 147.

25. Thompson RJ, Doran JF, Jackson P, Dhillon AP, Rode J. PGP. 9.5-a new marker for vertebrate neurons and neuroendocrine cells. Brain Res 1983; 278: 224

26. Fullen DR, Reed JA, Finnerty B, McNutt NS. S100A6 expression in fibrohistiocytic lesions. J Cutan Pathol 2001; 28: 229. 